\title{
Railway vehicle and bridge interaction: some approaches and applications
}

\author{
G. Mikheev, E. Krugovova \& R. Kovalev \\ Laboratory of Computational Mechanics \\ Bryansk State Technical University, Russia
}

\begin{abstract}
The present paper describes the CAE-based approach for analysis of dynamics of a coupled model of a flexible railway bridge and a train. The approach is being implemented in Universal Mechanism (UM) software. The railway bridge is considered as a flexible multibody system. The dynamics of flexible bodies are simulated using data imported from finite element analysis (FEA) software. An application of the approach to the investigation of dynamics of a railway vehicle and a bridge supposes taking into account the flexibility of the bridge. Comparison of flexible deflections and stresses for the full and reduced FE-models for static and moving loads are presented. The simulation results for a high-speed train on a bridge that is modelled as a reduced FE-model with 50, 100 and 200 flexible modes, as well as comparison of simulation results for separate and coupled approaches to vehicle-bridge interaction (VBI), are shown.
\end{abstract}

Keywords: vehicle-bridge interaction, flexible bridge model, moving load.

\section{Introduction}

Computer simulation is an effective approach to analyze the dynamics of railway bridges under train motion along them [1-4]. The main object of investigations can be both a bridge and a railway vehicle. From the point of view of bridges, purposes of researches could be the detection of resonance phenomena on railway bridges, dangerous operation conditions such as train speed and weight, specific bridge design and so on. As for high speed trains, a dynamic analysis is necessary because of resonance phenomena of the structures due to regularly spaced axle groups of the train. In the case of resonance, excessive bridge deck 
vibration can cause loss of wheel/rail contact, destabilization of the ballast and exceedance of the stress limits.

Analysis of the dynamics of the railway bridge and time histories of stresses and strains are required for the calculation of their durability. In this case, stress loading blocks are the results of dynamic simulation. These blocks are calculated based on time histories of bridge stresses obtained for selected modes of loading. The loading depends on the weight and speed of rail vehicles, track irregularities on the bridge and so on.

As for railway vehicle dynamics, it is important to consider the additional flexibility of the bridge in both vertical and lateral dynamics on safety, stability and ride comfort.

Usually, research of the dynamics of railway bridges is carried out based on a simplified description of the vehicle-bridge interaction. The widespread approach supposes analysis of a finite element model of a bridge under action of the moving loading that simulates a train. In most cases, constant values of forces that correspond to the weight distribution of the train vehicles are considered. Thus, the dynamics of the vehicles are not taken into account within the simplified approach. Besides, such models do not take into account the mutual influence between vehicles and bridges. It is their main disadvantage.

In this paper, methods for creating complex models, including a full 3D model of railway vehicles and trains and 3D models of flexible bridges, are considered. The methods are implemented in Universal Mechanism (UM) software [5].

\section{Equations of motion of a flexible body}

Equations of motion of a flexible body are derived using the floating frame of reference method (Shabana [6]). Linear flexible displacements of the body are described by the component mode synthesis method (Craig and Bampton [7] and Craig [8]). According to this method, flexible displacements are approximated by a sum of modes:

$$
\mathbf{u}=\sum_{j} \mathbf{h}_{j} w_{j}=\mathbf{H} \mathbf{w},
$$

where $\mathbf{u}$ is a matrix-column of nodal degrees of freedom of the flexible body, $\mathbf{h}_{\mathrm{j}}$ is a matrix-column of the mode and $w_{j}$ is a modal coordinate that defines flexible displacements corresponding to the mode with number $j$.

Modes of flexible body are calculated by an external FEA program and imported to UM software. A subsystem technique is used for including a flexible body into a multibody system. The idea that flexible displacements of a body can be represented by the sum of a number of mode shapes, scaled by modal coordinates, can be extended to stresses in the body as well. Modal coordinates can be used as the scaling factors on the stress solution of each mode shape and the superposition of these scaled stresses represents the body's stress state instantaneously. 


\section{Simulation of a rail-bridge interaction}

Rail-to-wheel contact forces are applied to a flexible structure as a running load. Both lateral and vertical force components are considered. A flexible body interacts with other bodies of a multibody system via joints and force elements. Joint points and points of attaching force elements are usually located at nodes of a FE-model of the flexible body. Such an approach cannot be applied to simulation of an interaction of a railway vehicle with the flexible bridge, because loads move and act between nodes of the FE-mesh of the bridge.

In UM software, a rail is modelled as a massless visco-elastic force element (see Figure 1). Transversal $\Delta y_{r}$ and vertical $\Delta z_{r}$ deflections of the rail, as well as their time derivatives, depend on the position and velocity of point $\mathrm{K}$ of the force element attachment to ground. Rotation of the rail around a longitudinal axis is not taken into account.

If a rail lies on a flexible bridge, total rail stiffness is obtained as a sum of stiffness between the rail and the bridge (sleepers, roadbed and so on) and stiffness of the flexible bridge itself, Figure 2. During integration of equations of motion, a position of point $\mathrm{K}$ is defined by the current position of a wheel.

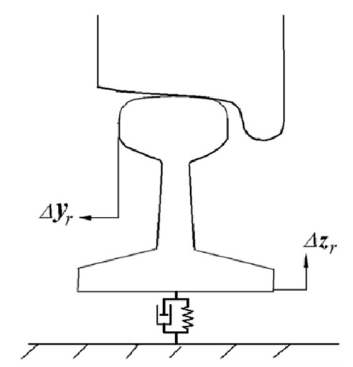

Figure 1: $\quad$ Massless model of a rail.
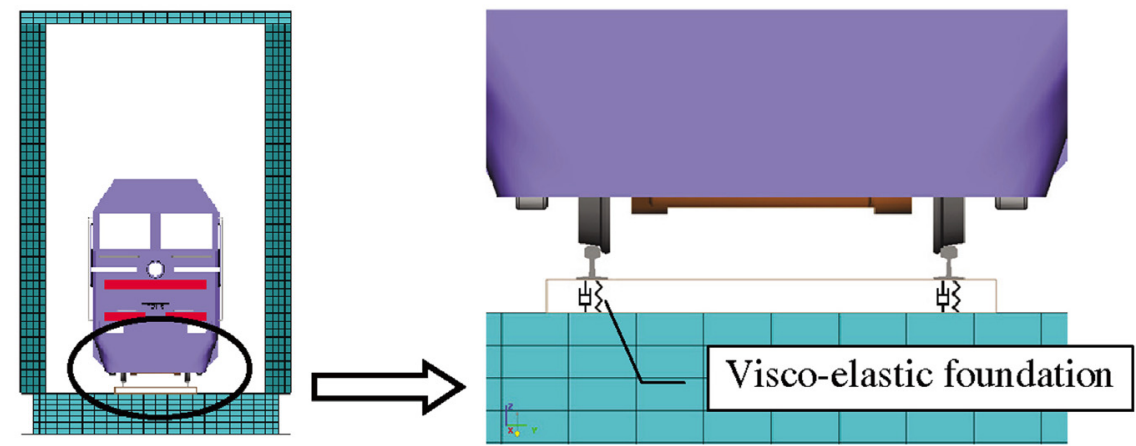

Figure 2: $\quad$ Model of interaction of a railway vehicle with a flexible bridge. 

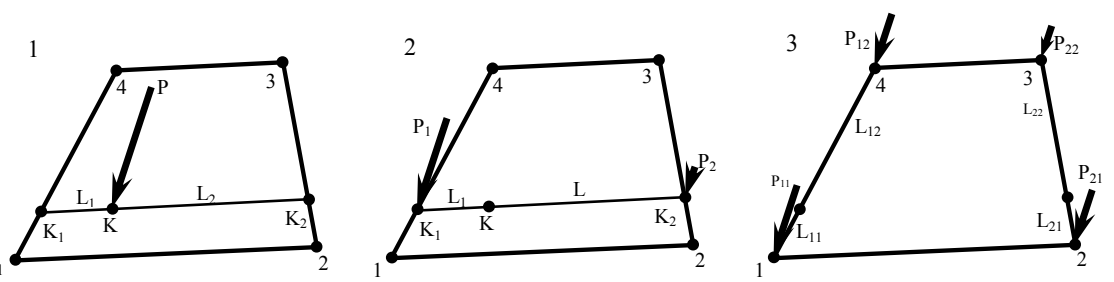

Figure 3: Simple algorithm of nodal force decomposition.

Therefore, it is necessary to compute the position and velocity of any point on the surface of the flexible bridge, as well as to apply calculated force to any point on a surface of the bridge.

Since the FEA-approach supposes force applying to a flexible body in nodes of FE-mesh only, the simple algorithm of the decomposition of wheel-to-rail contact forces between the nearest nodes of FE-mesh was used. Let us consider the algorithm in detail. A control area consists of surface polygons of a finite element model of the bridge, which is created around a rail-wheel contact point. The position and velocity of point $\mathrm{K}$ are calculated as linear interpolation of the corresponding values of the nearest nodes. Interaction forces are also distributed between the nearest nodes, Figure 3.

\section{Separate and coupled approaches to simulate VBI}

Let us discuss two typical approaches for the analysis of vehicle-bridge interaction and the stress-strain state of a bridge.

The so-called separate approach is the typical one that is used in many papers. It supposes considering a dynamical model of a railway vehicle and a model of a bridge separately. It means that wheel-to-rail contact forces are obtained from simulation of a railway vehicle without taking into account vehicle-bridge interaction. As a result, the dynamical analysis time histories of contact forces are saved. Then the obtained wheel-to-rail contact forces are applied to the FE-model of a bridge as running loads at the points that correspond to the positions of the wheels, Figure 4. Since vehicle dynamics is simulated without any reference to a bridge, the separate approach cannot give us any vehiclerelated performances, such as safety, stability or ride comfort, that would describe exactly vehicle-bridge interaction. So the separate approach can be used as a good approximation for the bridge response, but it is completely useless with regard to obtaining the vehicle dynamical response to running through the bridge.

The so-called coupled approach supposes the mutual vehicle-bridge dynamics, Figure 5. Total displacements of rails are obtained as a sum of displacements between the rail and the bridge due to sleepers and roadbed and flexible displacements of the bridge itself. The obtained total displacements finally influence the contact wheel-to-rail forces that in fact act on wheelsets and the bridge and thereby couple vehicle and bridge dynamics. So the coupled approach connects vehicle and bridge models in the integrated model and 
provides results concerning not only the bridge, but also vehicle response on vehicle-bridge interaction, which can also be the focus of applied researches.

\section{Applications}

\subsection{Verification of reduced FE-model: static test}

Since the component mode synthesis method supposes significant reduction of degrees of freedom of the flexible bodies, then compare results of static calculations with the full FE-model of the bridge in NASTRAN [9] and reduced FE-model in the Universal Mechanism software.

Let us consider the model that is depicted in Figure 6. A locomotive of 138,4 tonns stands in the middle of the first span of the bridge. The locomotive has 6 wheelsets. So, locomotive static load can be modelled as 12 lumped forces of $113142 \mathrm{~N}$ that represent the wheel loads.
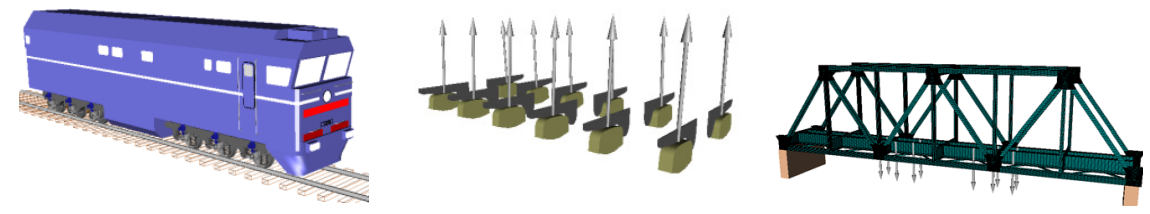

a) Vehicle dynamics

b) Obtaining contact forces c) Applying moving load

Figure 4: $\quad$ Separate approach.

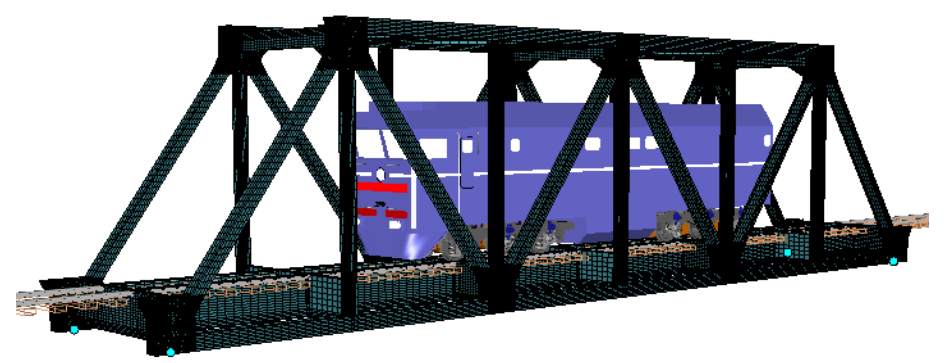

Figure 5: Coupled approach.
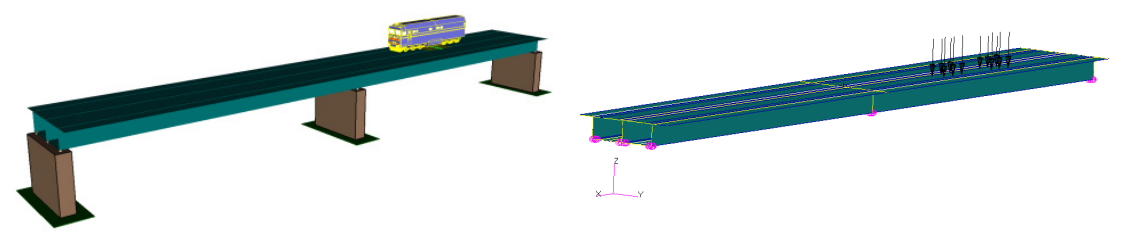

Figure 6: Single locomotive on a bridge: computer model and design diagram. 
Nine interface nodes in places of attachments of spans to piers (three nodes per pier) were chosen according to the component mode synthesis method. The flexibility of the piers was not taken into account.

The model of the bridge includes 40902 nodes and 45200 shell and beam finite elements. The full model has nearly 245400 d.o.f. The reduced model has 98 d.o.f. The cross section of the bridge is presented in Figure 7. Some of the flexible modes used, which are the result of orthonormalization of the component modes, are shown in Figure 8. Eight control nodes were chosen to compare results for the full and reduced FE-models of the flexible bridge, Figure 9.

The comparative simulation results are given in Table 1. It is clearly shown that the relative error is less than $2 \%$ for flexible deflection, and less than $5 \%$ for stresses.

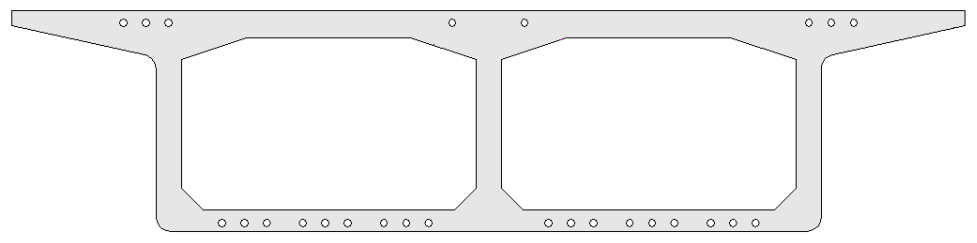

Figure 7: Cross section of a two-way bridge.

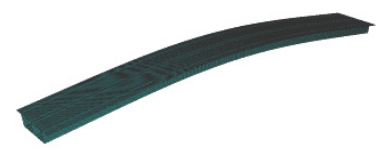

$1.65 \mathrm{~Hz}$

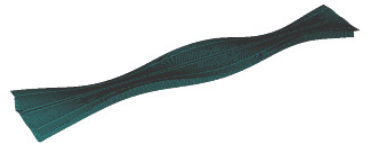

$11.5 \mathrm{~Hz}$

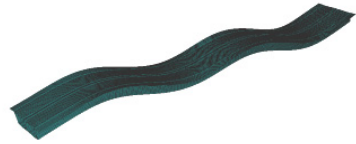

$11.8 \mathrm{~Hz}$

Figure 8: $\quad$ Some flexible modes of the bridge.

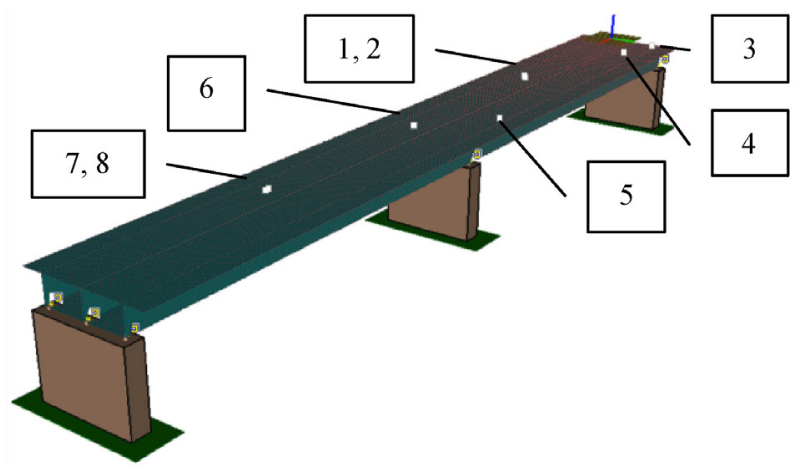

Figure 9: Control points on the bridge. 
Table 1: $\quad$ Node deflections and stresses.

\begin{tabular}{|c|c|c|c|c|c|c|}
\hline \multirow{2}{*}{ Node } & \multicolumn{2}{|c|}{ Deflection, mm } & \multicolumn{2}{c|}{$\begin{array}{c}\text { Longitudinal stress, } \\
\text { MPa }\end{array}$} & \multicolumn{2}{c|}{ Relative error, \% } \\
\cline { 2 - 7 } & $\begin{array}{c}\text { Reduced } \\
\text { FE-model }\end{array}$ & $\begin{array}{c}\text { Full } \\
\text { FE-model }\end{array}$ & $\begin{array}{c}\text { Reduced } \\
\text { FE-model }\end{array}$ & $\begin{array}{c}\text { Full } \\
\text { FE-model }\end{array}$ & Deflection & Stress \\
\hline 1 & -23.10 & -23.10 & -1.88 & -1.91 & 0 & 1.57 \\
\hline 2 & -23.20 & -23.20 & -1.92 & -1.96 & 0 & 2.04 \\
\hline 3 & -1.28 & -1.30 & -0.17 & -0.17 & 1.54 & 0 \\
\hline 4 & -1.09 & -1.09 & -1.62 & -1.61 & 0 & 0.62 \\
\hline 5 & -8.30 & -8.30 & 1.23 & 1.23 & 0 & 0 \\
\hline 6 & -1.07 & -1.09 & 2.03 & 2.05 & 1.83 & 0.98 \\
\hline 7 & -18.10 & -18.10 & -1.43 & -1.44 & 0 & 0.69 \\
\hline 8 & -18.10 & -18.20 & -1.45 & -1.47 & 0.55 & 1.36 \\
\hline
\end{tabular}

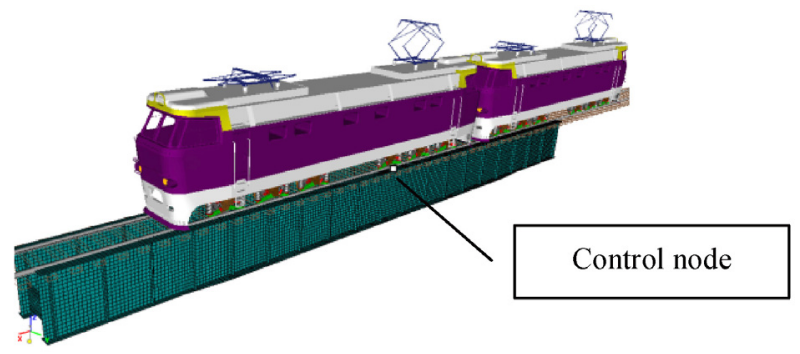

Figure 10: Computer model for the moving load test.

\subsection{Verification of the reduced FE-model: moving load test}

The second verification of the reduced FE-model of the bridge was done for the moving load. The model consists of a two-section electric locomotive on a flexible bridge, Figure 10. The FE-model of the bridge consists of 17907 nodes and 17641 beam and shell finite elements. The full FE-model has more than 105000 d.o.f. and the reduced one has only 200 d.o.f. The vehicle velocity is $80 \mathrm{~km} / \mathrm{h}$. Irregularities of the railway track were ignored.

A comparison of the flexible deflections for the moving load for the full FEmodel of the bridge in MIDAS software [10] and the reduced FE-model of the bridge in UM software is given in Figure 11. Flexible deflections due to a moving load (without deflection due to the weight of the bridge itself) in the middle of the span in the upper chord of the bridge are given. As is presented in Figure 11, flexible deflections for the full and reduced FE-models of the bridge are nearly the same. This means that reduced models of the bridge can be successfully used for the simulation of vehicle-bridge interaction within multibody system dynamics simulation codes. 


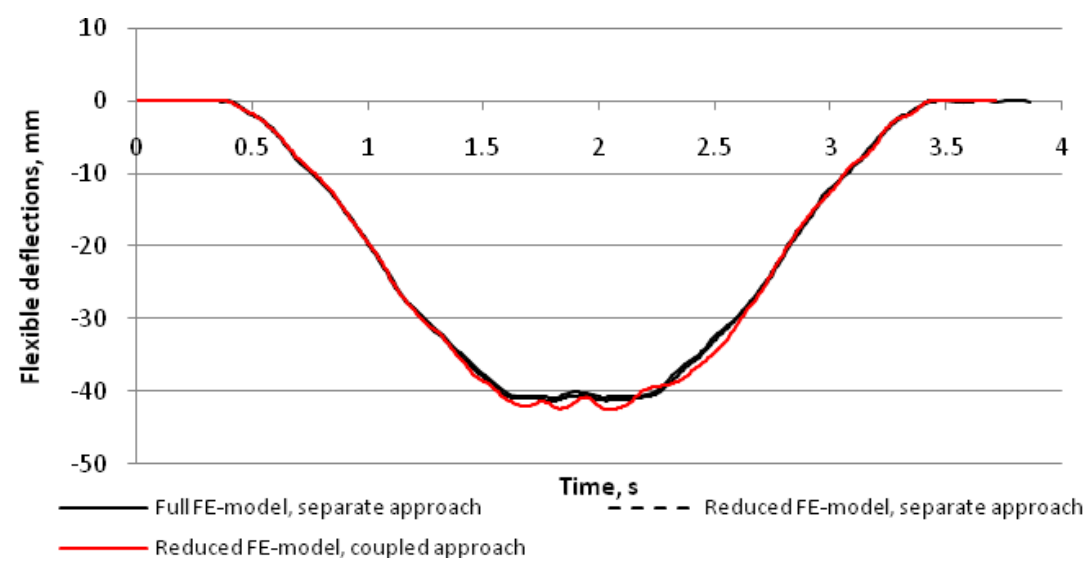

Figure 11: Results for moving load test: full and reduced flexible models.

\subsection{Trains on the bridge}

Let us consider the two examples of the simulation of the motion of trains on a bridge. The examples are focused on highlighting the following questions. The first one is how many flexible modes of a bridge should be taken into account to obtain more or less accurate results. The second one is how great an effect the mutual vehicle-bridge interaction has. Is it really necessary to consider significantly more complex mutual models or is it enough to represent a vehicle simply as a moving load according to the separate approach?

UM software supports the simulation of railway vehicles of all types: diesel and electric locomotives, freight and passenger cars, trains and special railway vehicles. Dynamic models of railway vehicles undergo no simplifications for VBI simulation in comparison with usual vehicle dynamics analysis. So, simulation results related to vehicles can be considered as valid and accurate enough. The correctness of mathematical models and used numerical algorithms in Universal Mechanism software were proven by the Manchester benchmarks (Iwnicki [11], Universal Mechanism [12]). Railway vehicles can be simulated as rigid or rigid-flexible multibody systems.

A high-speed passenger train and a heavy-haul train on the same bridge will be considered below. The model of the bridge that is described in Section 5.1 was used, see Figures 7-9.

\subsubsection{High-speed train}

The high-speed train consists of 10 vehicles (totally 294 d.o.f.), Figure 12 . The train is modelled as a typical multibody system and runs at $200 \mathrm{~km} / \mathrm{h}$. Let us compare some results for the flexible bridge described with the help of 50, 100 and 200 fixed interface eigenmodes calculated according to the component mode synthesis method. 


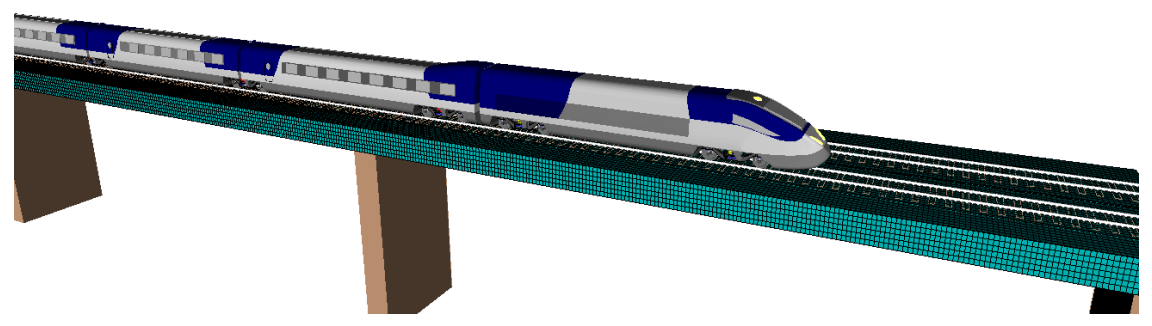

Figure 12: Simulation of high-speed train motion on the flexible bridge.

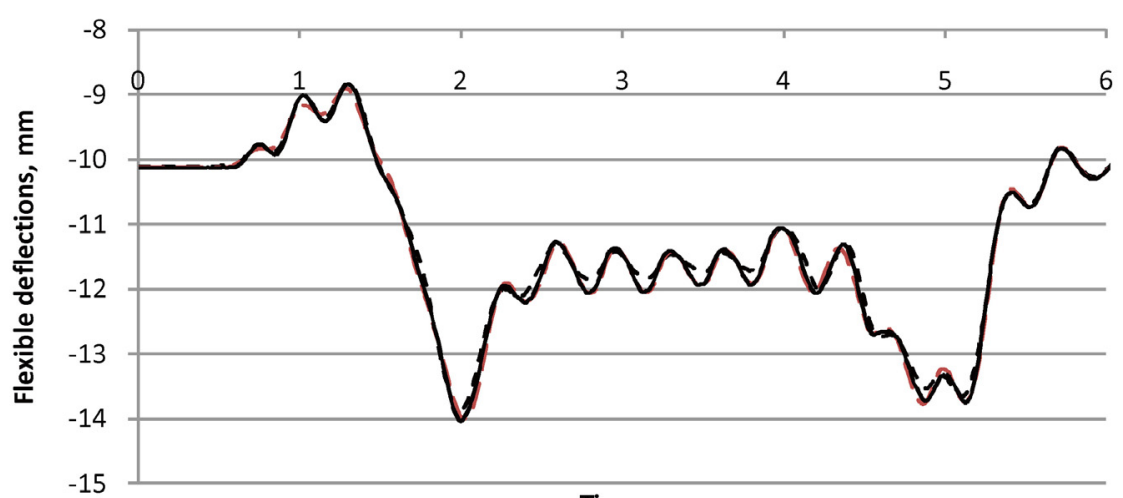

Time, $\mathbf{s}$

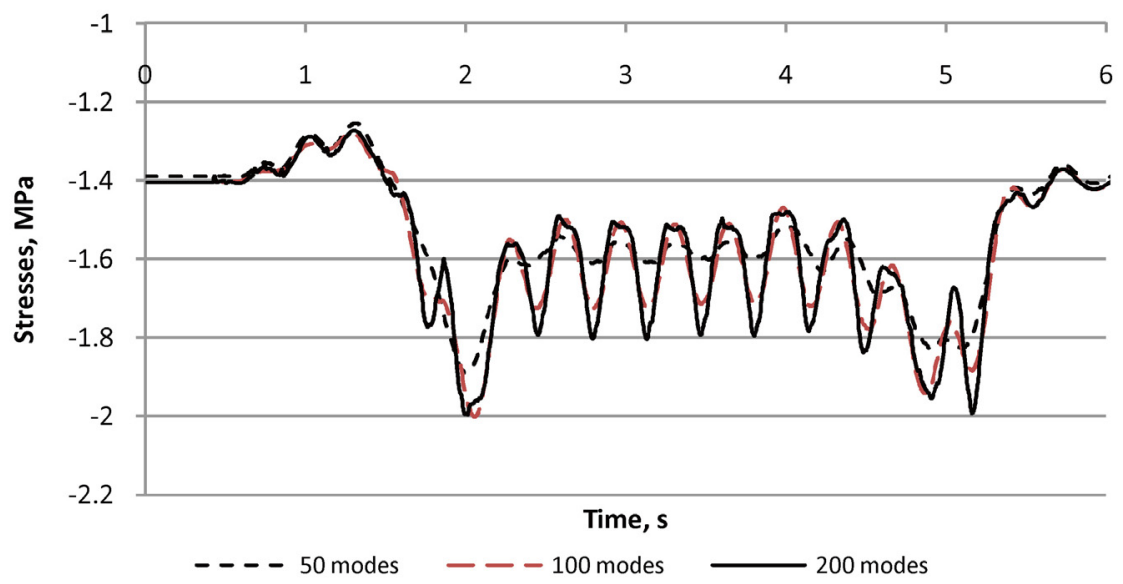

Figure 13: Flexible deflections and stresses for 50, 100 and 200 fixed interface eigenmodes, coupled approach.

Flexible deflections and stresses for the node in the middle of the first span of the bridge are shown in Figure 13. All models include 54 constraint modes and from 50 up to 200 fixed interface eigenmodes. It is obvious that the model with 200 eigenmodes is the most accurate one. 


\subsubsection{Heavy-haul train}

A model of a heavy-haul train consists of 2 locomotives and 8 freight cars (totally 892 d.o.f.), Figure 15 . The train runs at $100 \mathrm{~km} / \mathrm{h}$. Let us compare the obtained flexible deflections and stresses for the flexible bridge (200 fixed interface eigenmodes for the flexible bridge were used) for the separate and coupled approaches, see Figures 16 and 17.

The comparison of results for the separate and coupled approaches is presented in Figures 14, 16 and 17 and shows that there is no significant difference between the simulation results for the mentioned approaches. Please note that the difference for the separate and coupled approaches is larger for the heavier heavy-haul train despite its speed being two times smaller. Please note that the considered models include the relatively stiff steel concrete bridge. The difference between the results of the separate and coupled approaches might be more evident for other bridges of different design.

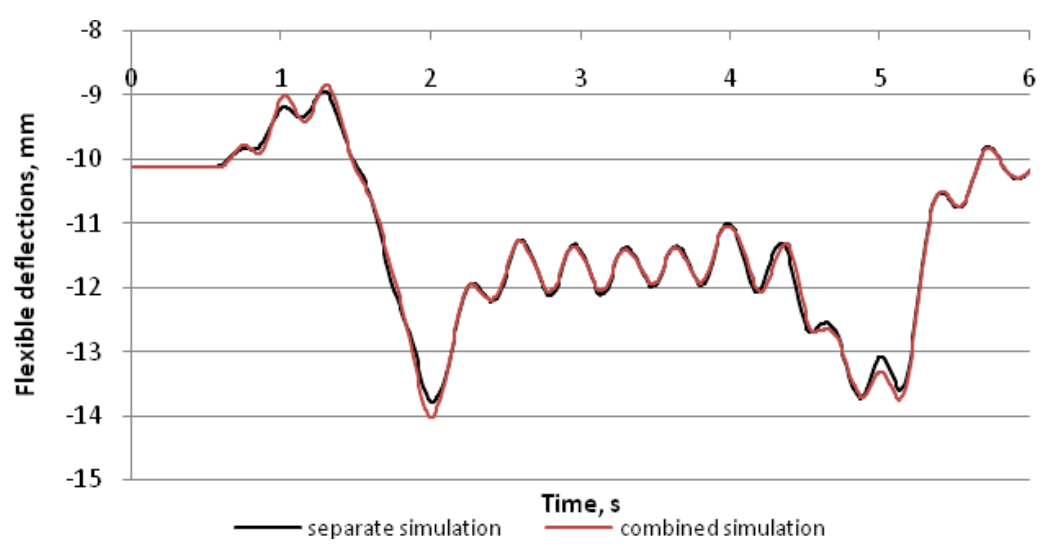

Figure 14: Flexible deflections for the separate and coupled simulations.

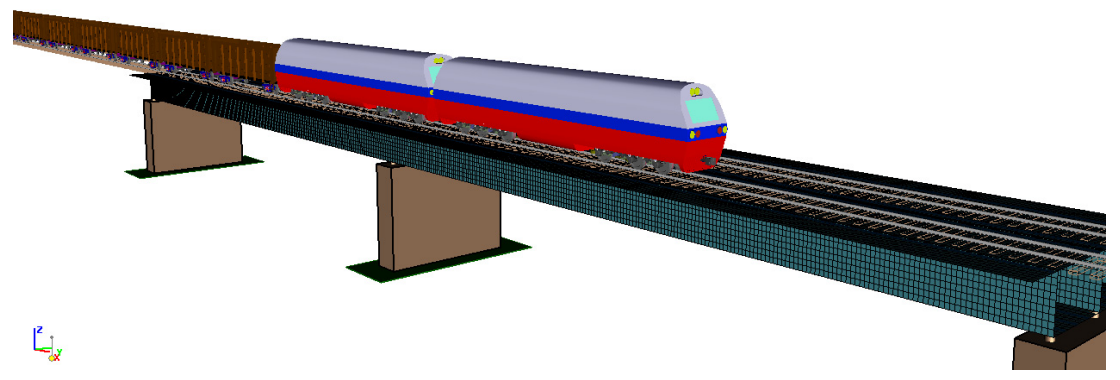

Figure 15: Simulation of heavy-haul train motion on the flexible bridge. 


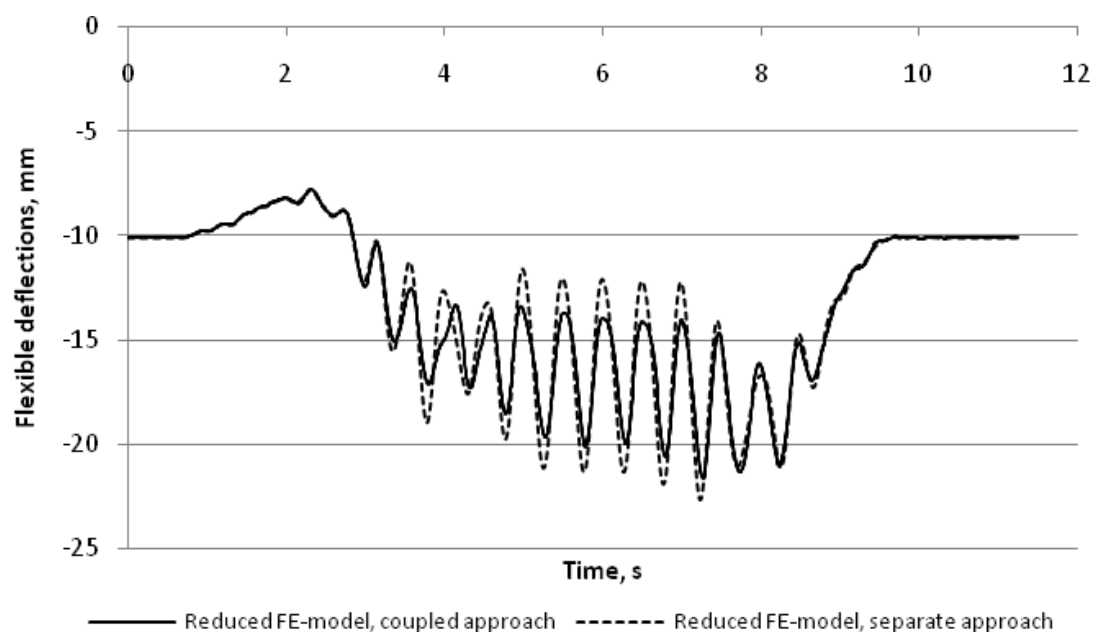

Figure 16: Flexible deflections for the separate and coupled simulations.

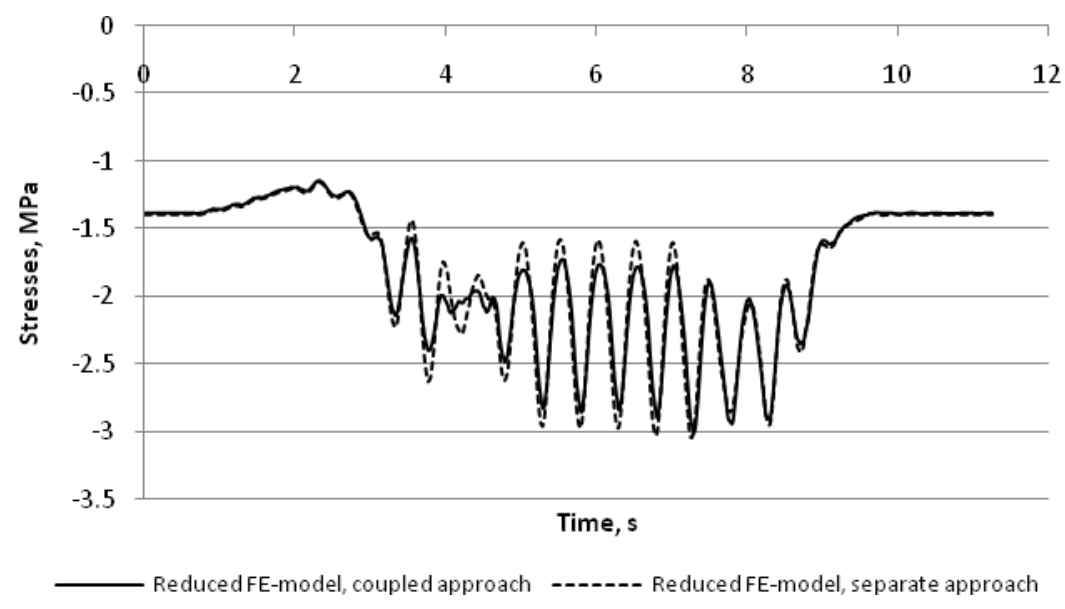

Figure 17: Flexible stresses for the separate and coupled simulations.

\section{Conclusions}

Reduced FE-models of bridges show generally very good agreement with the full FE-models for static test. The relative error between full and reduced FE-models is less than $2 \%$ for flexible displacements and less than $5 \%$ for stresses.

Simulation results for the moving load test also showed a very good agreement between full and reduced FE-models that proved the practical possibility of using reduced FE-models of bridges within software codes for the simulation of vehicle-bridge interaction. 
A different number of considered flexible modes for a FE-model of a bridge (50, 100 and 200 d.o.f.) expectedly influences both flexible deflections and stresses. The maximum difference is obtained for stress amplitudes.

The considered model of the high-speed and heavy-haul trains on the bridge did not show any significant difference between the separate and coupled approaches for simulating a vehicle-bridge interaction.

The proposed approach proved to be an effective tool for a detailed analysis of the vehicle-bridge interaction, taking into account the flexibility of the bridge.

\section{Acknowledgements}

This research is supported by the Russian Foundation for Basic Researches under grant no 08-01-00677-a.

Simulation results for the moving load test with the full FE-model (Section 5.2) in MIDAS software were kindly provided by Eng. Mikhail Malgin, The Paton Electric Welding Institute, Kiev, Ukraine.

\section{References}

[1] Gong, L. \& Cheung, M. S., Computer simulation of dynamic interactions between vehicle and long span box girder bridges. Tsinghua Science and Technology, Volume 13, Number 81, 2008.

[2] Xia, H., Zhang, N.\& De Roeck, G., Dynamic analysis of high speed railway bridge under articulated trains. Computers and Structures, 81, pp. 2467-2478, 2003.

[3] Yang, Y. B., Yau, J. D., \& Wu, Y. S. Vehicle-Bridge Interaction Dynamics, World Scientific Publishing Co. Pte. Ltd., 2004.

[4] Gong, L. \& Cheung, M. S., Computer simulation of dynamic interactions between vehicle and long span box girder bridges. Tsinghua Science and Technology, Volume 13, Number 81, 2008.

[5] Universal Mechanism software, http://www.umlab.ru

[6] A.A. Shabana. Flexible multibody dynamics: review of past and recent developments. Multibody System Dynamics, 1, pp. 189-222, 1997.

[7] Craig, R.R. Jr. \& Bampton, M.C.C., Coupling of substructures for dynamic analysis. AIAA Journal, Vol. 6, No. 7, pp. 1313-1319, 1968.

[8] Craig, R.R. Jr., Coupling of substructures for dynamic analysis: an overview. In AIAA Paper, No 2000-1573, AIAA Dynamics Specialists Conference, Atlanta, GA, April 5, 2000.

[9] MSC.NASTRAN, http://www.mscsoftware.com

[10] MIDAS Family Programs, http://www.midasuser.com

[11] Iwnicki, Simon D. The Manchester benchmarks for rail vehicle simulation / ed. by S. Iwnicki. - Lisse: Swets \& Zeitlinger, 1999.

[12] The Manchester benchmarks for rail vehicle simulation in Universal Mechanism software. http://www.umlab.ru/download.htm 